\title{
Fitness Consequences of Malathion-Specific Resistance in Red Flour Beetle (Coleoptera: Tenebrionidae) and Selection for Resistance in the Absence of Malathion
}

\author{
ERIC HAUBRUGE AND LUDOVIC ARNAUD
}

Unit of Pure and Applied Zoology - Gembloux Agricultural University, B-5030 Gembloux, Belgium

\begin{abstract}
Malathion resistance in the red flour beetle, Tribolium castaneum (Herbst), is a worldwide problem and is very stable once it becomes widespread in natural populations. In the absence of insecticide the proportion of resistant phenotypes may rapidly decline but the development of resistance does not always involve reduced fitness. Malathion-specific resistance in T. castaneum seems not to involve any loss of fitness in laboratory or field conditions. Susceptible beetles were in competition with resistant beetles at different initial frequencies and modifications of susceptible gene frequency were estimated in these laboratory populations over 10 generations. A significant decrease in susceptible gene frequency was observed in Tribolium populations over time. The selection coefficient of the susceptible allele was estimated and the fitness of susceptible alleles in all tests was observed to range from 0.89 to 0.93 compared with the fitness of resistant genotypes, which was assumed to be 1. Data provided evidence that the resistant strains exhibited fitness advantages in the absence of malathion. We also compared the biotic potential (fecundity and developmental time) of the susceptible strain, the homozygous malathion-specific resistant strain, and their hybrids. Malathion-specific resistant strains showed an $8-23 \%$ increase in biotic potential relative to the susceptible strain. These findings are consistent with those of malathion-specific resistance in T. castaneum; the fitness of the insects seems independent of the genetic background and the fitness of the resistant insects is not affected by this resistance mechanism.
\end{abstract}

KEYWORDS : Tribolium castaneum, Coleoptera, insecticide, malathion resistance, fitness, selection

Because of the intensive use of malathion in grain storage and on stored grain since the late 1950s, mal-athionresistance in red flour beetle, Tribolium castaneum (Herbst), is widespread. The first case of resistance was reported in 1961 (Parkin et al. 1962), and by 1974 this phenomenon was regarded as a common attribute of this species (Champ and Dyte 1976). Although malathion use has declined or even been abandoned in some countries from the early 1970s because of the widespread occurrence of malathion resistance in red flour beetle populations (Champ 1984), malathion-specific resistance is very stable in T. castaneum wild populations. Moreover, the malathion-specific resistant phenotype in T. castaneum populations has almost completely replaced the susceptible one throughout most of the world (Beeman and Nanis 1986). To explain this stability, it was assumed that there were no pleiotropic effects of the mutation and no or few reproductive disadvantages between malathion-specific resistant and susceptible strains.

Because resistant insects were not present at high frequency before the use of insecticides, it is not surprising that resistant and susceptible strains should differ in properties other than their adaptation to insecticides, such as developmental time, fecundity, and fertility. Differences in the biological parameters affecting the net reproductive rate and the innate capacity of insect populations to increase are of particular interest to insecticide resistance management. Although the majority of fitness studies has shown that there are fitness costs associated with insecticide resistance (Ferrari and Georghiou 1981, Argentine et al. 1989, Parello and Trumble 1989, White and Bell 1990, Cochran 1993, McKenzie 1994), in some cases, in the absence of treatment, there is no fitness difference between resistant and susceptible strains or the resistant strain has a fitness advantage (Roush and Hoy 1981, Beeman and Nanis 1986, McKenzie 1993, Spollen et al. 1995, White and Bell 1995).

In this study, we evaluated fitness consequences of malathion resistance in $T$. castaneum to understand why persistence of this resistance mechanism occurs in wild populations. We compared the biotic potential $\left(\mathrm{B}_{\mathrm{P}}\right)$ of susceptible and malathion-specific resistant strains of T. castaneum. With this information, we can better assess the likelihood of insecticide resistance increasing to cause widespread control failures against stored-product pests. 


\section{Materials and Methods}

Strains. Two red flour beetle strains were used in this study. A strain specifically resistant to malathion, called PRm, was originally collected from a grain store in the Philippines in 1976. The other strain, Asm, is susceptible to malathion and originated from storage facilities at Abidjan, Ivory Coast, in 1984. The beetles were reared on whole wheat flour enriched with brewer's yeast (10:1 wt:wt) and kept in the dark at $30 \pm 3{ }^{\circ} \mathrm{C}$ and $65 \pm 5 \% \mathrm{RH}$. The PRm strain was exposed to a continuous dose of malathion $\left(148 \mu \mathrm{g} / \mathrm{cm}^{2}\right.$ on filter paper) for 37 generations and was selected for homozygous malathion resistance by exposure malathion $\left(14.8 \mu \mathrm{g} / \mathrm{cm}^{2}\right.$ on filter paper $)$ for a discriminating time of $3 \mathrm{~h}$. The homozygous susceptible Asm strain was maintained without pesticide exposure for 8 yr. Separate cultures were started with homozygous resistant PRm eggs and homozygous susceptible Asm eggs and heterozygous eggs laid by the susceptible female parents (PRm male x Asm female) or by the resistant female parents (Asm male x PRm female).

Malathion Susceptibility and Dominance of Resistance. Adult beetles (2-4 wk old) were tested for malathion resistance with an insecticide contact bio-assay. For each concentration tested, 100 adults were exposed for $24 \mathrm{~h}$ to a filter paper impregnated with an acetonic solution of malathion $(943 \mu \mathrm{l})$ at concentrations ranging from 0 to $30 \%$ (wt:vol) at $25^{\circ} \mathrm{C}$. Controls were exposed to acetone-impregnated papers. Afterward, the number of dead adults was observed. We considered adults to be dead when the beetles were motionless or exhibited completely uncoordinated movements. Mortality was determined $24 \mathrm{~h}$ later and compared with controls that were treated with malathion only. Data were pooled, and $\mathrm{LC}_{50}, \mathrm{LC}_{90}$, and their corresponding resistance factors were determined by Logit-Probit regression analysis (Raymond 1993). Dominance levels were measured as $D=\left(R_{F 1}\right.$ 1)/( $\left.R_{R}-1\right)$, where $R_{R}$ and $R_{F 1}$ are the resistance factors at $L C_{50}$; and $R_{R}$ and $R_{F 1}$ are defined by $L_{R} / L_{S}$ and $\mathrm{LC}_{\mathrm{F} 1} / \mathrm{LC}_{\mathrm{S}}$, respectively, where $\mathrm{LC}_{\mathrm{R}}, \mathrm{LC}_{\mathrm{S}}$, and $\mathrm{LC}_{\mathrm{F} 1}$ are the insecticide concentrations (in percent) needed to obtain 50\% mortality for homozygous resistant, homozygous susceptible, and heterozygous individuals (Bourguet et al. 1997).

Selection for Malathion-Specific Resistance in Absence of Insecticide. To assess the relative fitness of resistance allele(s) in absence of selective pressure, resistant phenotypes were placed in competition with susceptible insects without insecticide. The frequencies of the resistant and susceptible phenotypes were monitored for 10 consecutive, nonoverlapping generations. Population cages were made of 900-ml glass jars containing whole wheat flour enriched with brewer's yeast (10:1 wt:wt). The initial frequency of resistant allele was set at $0.05,0.10,0.20$, or 0.30 at a Hardy-Weinberg equilibrium by adding $R^{\text {mal }} / R^{\text {mal }}, R^{\text {mal }} / S^{+}$, or $S^{+} / S^{+}$beetles in the proper ratios, where $R^{\text {mal }}$ represents the allele of malathion-specific resistance and $\mathrm{S}^{+}$of insecticide susceptibility. Six independent lines were monitored for each initial $R^{\text {mal }}$ frequencies. To ensure nonoverlapping generations, parent beetles were discarded after $3 \mathrm{wk}$, which is less than the immature developmental period. After $8 \mathrm{wk}$, the progeny were collected from every jar. One hundred adults were randomly selected and used to initiate the next generation. In addition, $F_{l}$ adult progeny of each population were tested for malathion susceptibility as describe by Haubruge et al. (1997). Three replicates of 200 insects were confined on a filter paper impregnated with a $1 \%$ malathion concentration $\left(14.8 \mu \mathrm{g} / \mathrm{cm}^{2}\right)$ for $3 \mathrm{~h}$ at $25^{\circ} \mathrm{C}$ This discriminating dose was 10 times the $\mathrm{LC}_{99}$ of the susceptible strain Asm, but did not kill any of the resistant heterozygotes and homo zygotes.

Table 1. Expected frequencies of the different genotypes of susceptible homozygous, resistant homozygous or heterozygous and their fitness

\begin{tabular}{|l|c|c|c|}
\hline Genotype & $R^{\text {mal }} / R^{\text {mal }}$ & $R^{m a l} / S^{+}$ & $S^{+} / S^{+}$ \\
\hline Frequency & $\mathrm{p}^{2}$ & $2 \mathrm{pq}$ & $\mathrm{q}^{2}$ \\
\hline Fitness $(w)$ & $W_{R R}=1$ & $W_{R S}=1$ & $w_{\mathrm{ss}}=1-\mathrm{C}_{\mathrm{s}}$ \\
\hline
\end{tabular}

$\mathrm{C}_{\mathrm{s}}, \mathrm{p}$, and $\mathrm{q}$ are the selection coefficient, the frequency of resistant, and the frequency of susceptible alleles, respectively.

Estimates of Selection Coefficients and Relative Fitness. The frequencies of the different genotypes and their fitness can be summarized at Table 1 . If the malathion-specific resistance is inherited as a single dominant or codominant gene (see Results) and the frequency of susceptible genotype $\left(S^{+} / S^{+}\right)$decreased in Tribolium populations, the fitness of the resistant homozygous $\left(w_{R R}\right)$ and heterozygous $\left(w_{R S}\right)$ were assumed to be 1.0. The fitness of susceptible homozygous $\left(w_{s s}\right)$ was assumed to be $1-\mathrm{C}_{\mathrm{s}}$. 
The fitness of the susceptible genotype compared with the resistant genotype over 10 generations may be estimated from the change in frequency of that genotype during this time. The method of Clarke and Murray (1962) is appropriate when the gene frequency change is low as it is here (Muggleton 1986). Assuming the existence of a Hardy-Weinberg equilibrium among the tested Tribolium populations, estimates of gene frequencies $q_{0}$ and $q_{n}$ at the beginning and after each generation (n) were obtained. A simple expression is available for the change in $q$ as a result of one generation of selection: $\left[\Delta q=C_{s} \cdot q^{2}(1-q) / 1-C_{s}\left(1-q^{2}\right)\right]($ Falconer 1960). By integrating this over 10 generations an estimate of selection coefficient $\left(\mathrm{C}_{\mathrm{s}}\right)$ and his variance $\left[\mathrm{V}_{(\mathrm{Cs})}\right]$ could be calculated as (Clarke and Murray 1962):

$$
\begin{gathered}
C_{s}=\frac{\ln \left[\frac{q_{0}\left(I-q_{10}\right)}{q_{10}\left(I-q_{0}\right)}\right]+\frac{I}{q_{10}}-\frac{I}{q_{0}}}{t+\ln \frac{\left(I-q_{10}\right)}{\left(I-q_{0}\right)}} \\
V_{(C s)}=\left(\frac{I}{q_{10}}+\frac{I}{I-q_{10}}+\frac{I}{q_{10}^{2}}\right)^{2} \frac{q_{10}\left(1-q_{10}\right)}{2 N_{n}}+\left(\frac{I}{q_{0}}+\frac{I}{I-q_{0}}+\frac{I}{q_{0}^{2}}\right)^{2} \frac{q_{0}\left(I-q_{0}\right)}{2 N_{0}} /\left[t+\ln \left(\frac{I-q_{10}}{I-q_{0}}\right)\right]
\end{gathered}
$$

Egg Fertility and Biotic Potential $\left(\mathbf{B}_{\mathbf{P}}\right)$. The fertility of the eggs laid by homozygous and heterozygous females was estimated for the four strains. One hundred adults were placed in $90-\mathrm{mm}$ petri dish with $20 \mathrm{~g}$ of the rearing medium. Every $4 \mathrm{~d}$, the eggs laid during a 24 -d period were removed. One hundred eggs were randomly selected and placed in a 55-mm petri dish with $5 \mathrm{~g}$ of the rearing medium. After $6 \mathrm{~d}$, the number of hatched larvae was counted.

Both fecundity (F) and developmental time (DT) affect the reproductive potential of the insects. It is therefore interesting to pool these two parameters to compare the fitness of resistant homozygous and heterozygous strains to that of the susceptible strain. We used the biotic potential $\left(\mathrm{B}_{\mathrm{P}}\right)$ adapted from Roush and Plapp (1982) as follows:

$$
B_{P}=\ln F / D T_{r}
$$

where $\mathrm{F}$ is the fecundity (mean number of larvae/ female), and $\mathrm{DT}_{\mathrm{r}}$ is the ratio between the mean developmental time of the considered strain and that of the susceptible strain.

The fecundity and the ratio between the mean developmental time of the two homozygous strains (Asm and PRm) and those of their two reciprocal hybrids (PRm male x Asm female and Asm male x PRm female) were estimated and pooled to calculate the $\mathrm{B}_{\mathrm{P}}$ of the resistant insects.

To compare fecundity, pupae of every T. castaneum strain were sexed and maintained individually in a vial with the rearing medium to ensure the beetle virginity. Adult pairs consisting of a male marked with a black pencil and a female were placed at $30 \pm 3^{\circ} \mathrm{C}$ and $60 \pm 5 \% \mathrm{RH}$ in a $90-\mathrm{mm}$ petri dish with $20 \mathrm{~g}$ of the rearing medium. After $4 \mathrm{~d}$, the males were discarded and the females placed individually in a 55-mm petri dish with $5 \mathrm{~g}$ of the rearing medium. The females were allowed to lay eggs for $4 \mathrm{~d}$ and were discarded afterward. After 2 wk (a period long enough to allow all larvae to hatch under the rearing conditions), the number of larvae was counted in each dish. Fecundity studies were replicated 34 times for each strain. For developmental time estimates, 100 eggs laid during a $24-\mathrm{h}$ period were placed at $30 \pm 3{ }^{\circ} \mathrm{C}$ and $60 \pm 5 \% \mathrm{RH}$ in a $55-\mathrm{mm}$ petri dish with $20 \mathrm{~g}$ of the rearing medium. Three weeks later, emerging adults were counted at least once every day and developmental time means calculated. The experiments were replicated three times for each strain. The ratio between the mean developmental time of homozygous and heterozygous resistant strains were scaled against one of the susceptible strain Asm. $B_{p}$ was calculated on these scaled values, which we referred to as relative developmental time $\left(\mathbf{D T}_{\mathbf{r}}\right)$.

Statistical Analysis. The statistical analysis software Minitab version 12.2 for Windows (Minitab 1998) was used to analyze the results. One-way analysis of vari-anc (ANOVA) and Tukey's multiple comparison test were performed to compare the four strains (significant differences were considered when $\mathrm{P}$ values were $<0.05$ ). 
Table 2. Toxicity of malathion to strains of T. castaneum: Asm (susceptible), PRm (malathion-specific resistant), $\mathrm{H}_{1}$ (Asm male $x$ PRm female), and $\mathrm{H}_{2}$ (PRm male $x$ Asm female)

\begin{tabular}{|l|l|l|l|l|l|l|l|}
\hline Strain & $n$ & Slope \pm SE & $\mathrm{LC}_{50}$ & $95 \% \mathrm{CL}$ & $\chi^{2}$ & $\mathrm{df}$ & $\mathrm{RF}_{50}$ \\
\hline Asm & 100 & $14.35 \pm 1.08$ & 0.04 & $0.01-0.10$ & 6.79 & 5 & 1 \\
\hline $\mathrm{PRm}$ & 100 & $5.20 \pm 0.30$ & 8.61 & $7.54-12.41$ & 4.17 & 8 & 211.85 \\
\hline $\mathrm{H}_{1}$ & 100 & $3.28 \pm 0.29$ & 7.96 & $6.43-14.45$ & 3.49 & 3 & 174.78 \\
\hline $\mathrm{H}_{2}$ & 100 & $3.12 \pm 0.32$ & 9.12 & $6.78-13.89$ & 3.55 & 2 & 224.58 \\
\hline
\end{tabular}

$n$, Sample size per dose. RF50, resistance factor 50.

\section{Results}

Malathion Susceptibility of Strains and Dominance of Resistance. The malathion-specific resistant strain PRm was 212-fold resistant to malathion compared with the susceptible Asm strain at the $\mathrm{LC}_{50}$ (Table 2). The degree of dominance (Bourguet et al. 1997) was 0.9 and 1.0 for the heterozygous (PRm male x Asm female) strain and the heterozygous (Asm male x PRm female) strain, respectively. The reciprocal crosses gave almost identical results, confirming an autosomal mode of inheritance. Repeated backcross-ing of resistant hybrids to the Asm susceptible strain consistently yielded two offspring classes (resistant and susceptible) (data not shown). There was no segregation of the resistant class into partially resistant subclasses even after four consecutive generations of backcrossing. These observations provide evidence that resistance is primarily controlled by a single, dominant allele or closely linked alleles as previously reported (Beeman 1983, White and Bell 1988).

Selection for Malathion-Specific Resistance in the Absence of Insecticide: Selection Coefficients and Relative Fitness. Our results show that when resistant phenotypes are in competition with the susceptible T. castaneum phenotype, their frequency within populations increases slightly. After 10 generations, there is evidence of directed selection of the resistant allele because the mean frequency of the $\mathrm{S}^{+}$allele decreases under its original value in all initial frequencies tested (Fig. 1; Table 3). The selective advantage of $R^{\text {mal }}$ allele implied by the decline of the susceptible phenotype in the populations can be quantified by estimating the selection coefficient $\left(\mathrm{C}_{\mathrm{s}}\right)$ and the fitness $(w)$ of the different genotypes (Table 2). Although the fitnesses $\left(w_{R R}\right.$ and $\left.w_{R S}\right)$ are assumed to be 1.0, the fitness of susceptible homozygous $\left(w_{s s}\right)$ ranged from 0.891 to 0.930 after 10 generations for the different initial $R^{\text {mal }}$ frequencies. These data suggest that even in the absence of malathion, the malathionspecific resistant phenotypes of T. castaneum have a selective advantage compared with the susceptible phenotype.

Table 3. Selection coefficients and fitness of malathion susceptible genotype $\left(S+/ S^{+}\right)$ofT. castaneum in relation with the hiitial frequency of resistant allele $\left(R^{\mathrm{mal}}\right)$ over 10 consecutive nonover-lapping generations

\begin{tabular}{|l|l|l|l|l|l|}
\hline $\begin{array}{l}\text { Initial } R^{\text {mal }} \\
\text { Frequency }\end{array}$ & $\begin{array}{l}\text { Initial S } \\
\text { Frequency }\end{array}$ & $\begin{array}{l}\text { Final S } \\
\text { Frequency }\end{array}$ & $\begin{array}{l}\text { Selection } \\
\text { Coefficient }\end{array}$ & $\mathrm{V}_{(\mathrm{Cs})}{ }^{{ }^{+}}$ & $\boldsymbol{s}^{+} \mathbf{s}^{+}$fitness \\
\hline$\left(\mathrm{p}_{0}\right)$ & $\left(\mathbf{q}_{\mathbf{0}}\right)$ & $\left(\mathrm{q}_{10}\right)$ & $\left(\mathrm{C}_{\mathrm{S}}\right)$ & 0.00068 & 0.891 \\
\hline 0.05 & 0.95 & 0.83 & 0.109 & 0.00051 & 0.919 \\
\hline 0.10 & 0.90 & 0.78 & 0.081 & 0.00045 & 0.909 \\
\hline 0.20 & 0.80 & 0.63 & 0.091 & 0.00055 & 0.930 \\
\hline 0.30 & 0.70 & 0.57 & 0.070 & & \\
\hline
\end{tabular}

${ }^{a} \mathrm{~V}_{(\mathrm{Cs})}$, variance of selection coefficient.

${ }^{b} w_{s s}=1-\mathrm{C}_{\mathrm{s}}$ 


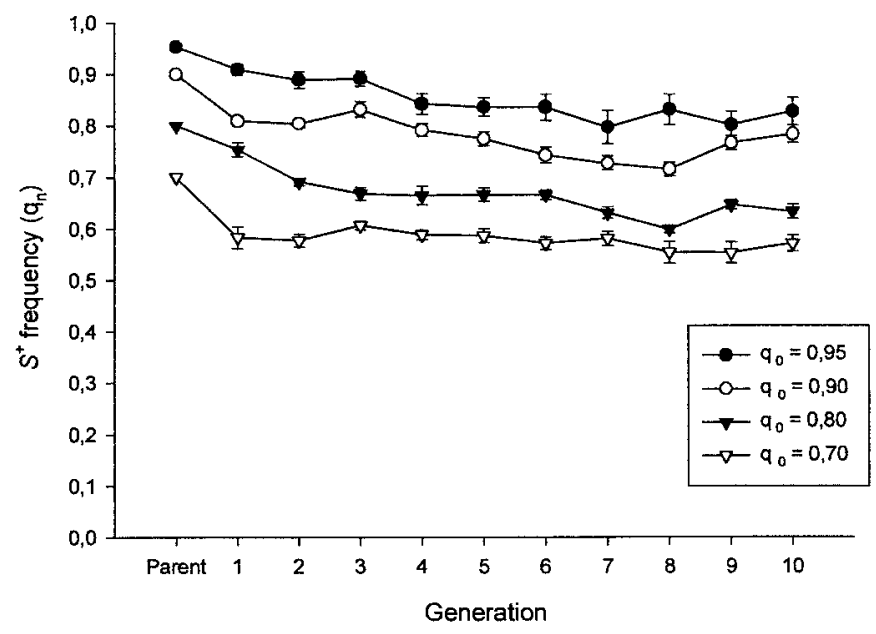

Fig. 1. Evolution of the frequency of malathion susceptible allele $\left(S^{+}\right)$in $T$. castaneum populations during 10 consecutive and nonoverlapping generations. Six independent lines were monitored for each of four initial $R^{\text {mal }}$ frequencies. Each symbol (mean $\pm S E$ ) shows the frequency of $S^{+}$allele in the population.

Egg Fertility and $\mathbf{B}_{\mathbf{p}}$. Egg fertility did not differ among the four strains (Table 4; one-way ANOVA, $F=0.03$; $\mathrm{df}=3,20 ; \mathrm{P}=0.994)$. There was a significant difference in fecundity of the females of the four strains (one-way ANOVA, $F=48.07 ; \mathrm{df}=3,132 ; \mathrm{P}<0.0001)$. The fecundity of the susceptible females was significantly lower than that of the females of the three resistant strains (Tukey's test, $\mathrm{P}<0.05$ ). Measurements of developmental time showed that DT of heterozygous resistant and susceptible strains was generally shorter than the DT of homozygous resistant strain. However, there is a significant difference between the DT of the four strains (oneway ANOVA, $\mathrm{F}=208.81 ; \mathrm{df}=3,893 ; \mathrm{P}<0.0001$ ) and the DT of the susceptible strain is the shortest (Tukey's test, $\mathrm{P}<0.05)$. For malathion resistance and fecundity, the ( $\mathrm{PRm}$ male $\mathrm{x}$ Asm female) heterozygous strain had a greater fitness advantage than the homozygous resistant strain with regard to DT, probably due to the heterosis effect (Table 4).

The $\mathrm{B}_{\mathrm{P}}$ of every strain is reported in Table 4. Mal-athion-specific resistance in T. castaneum does not involve any reduction of $\mathrm{B}_{\mathrm{P}}$. Moreover, the biotic potential of the resistant phenotypes is slightly higher than that of the susceptible strain.

Although we observed that heterozygous resistant insects were more fit than homozygous resistant, the ratio of their $\mathrm{B}_{\mathrm{p}}=\mathrm{B}_{\mathrm{p}} \mathrm{PRm} /\left(\left\{\mathrm{B}_{\mathrm{p}} \mathrm{Hl}+\mathrm{B}_{\mathrm{p}} \mathrm{H} 2\right\} / 2\right)$ was 1.11, this is not really in contradiction with the assumption made in the model to estimate the coefficient of selection $\left(\mathrm{C}_{\mathrm{s}}\right)$ of the $R^{\text {mal }}$ gene, where the fitness of the two heterozygous and the homozygous resistant insects was assumed to be equal.

\section{Discussion}

Malathion-Specific Resistance was Assessed Genetically in T. castaneum by Conventional Experiments.

Although Beeman (1983) determined that this resistance mechanism was inherited as a semidominant trait, we suggest that it is inherited as a monogenic autosomal dominant Mendelian trait. However, Wool et al. (1982) determined that it was inherited as a dominant or overdominant character. McKenzie and Whitten (1982) reported that pesticide resistance evolving in an agricultural environment is usually controlled by one or a few genes. Moreover, pleiotropic effects of insecticide resistance gene(s) on fitness traits are among the factors that could affect the persistence of resistance in a population of mixed genotypes (Georghiou 1983). It has been assumed that resistance gene(s) have fitness costs resulting in low frequency of resistant phenotypes before the population is in contact with the insecticide. Moreover, fitness values varying from 0.5 to 0.8 for resistant insects compared with susceptible insects have been observed in populations of mosquitoes (Ferrari and Georghiou 1981), blowflies (McKenzie and O'Farrell 1993), and beetles (Muggleton 1983) in the absence of insecticide. 
Table 4. Fertility, fecundity, developmental time, and biotic potential of T. castaneum strains: Asm (susceptible), PRm (malathion-specific resistant), $\mathrm{H}_{1}$ (Asm male $x$ PRm female), and $\mathrm{H}_{2}$ (PRm male $x$ Asm female)

\begin{tabular}{|l|c|c|c|c|c|c|c|c|c|c|}
\hline Strain & $n$ & Fertility (\%) & $n$ & $\mathrm{~F}$ & $\ln \mathrm{F}$ & $n$ & $\mathrm{DT}$ & $\mathrm{DT}_{\mathrm{r}}$ & $\mathrm{B}_{\mathrm{P}}$ & $\mathrm{CBP}$ \\
\hline Asm & 600 & $72.2 \pm 2.1$ & 34 & $33.9 \pm 3.3 \mathrm{a}$ & 3.52 & 206 & $29.8 \pm 0.1 \mathrm{a}$ & 1.00 & 3.52 & 1 \\
\hline PRm & 600 & $73.0 \pm 7.4$ & 34 & $71.8 \pm 2.6 \mathrm{bc}$ & 4.27 & 227 & $34.1 \pm 0.2 \mathrm{~d}$ & 1.13 & 3.79 & 1.08 \\
\hline $\mathrm{H}_{1}$ & 600 & $72.1 \pm 5.4$ & 34 & $65.5 \pm 2.4 \mathrm{~b}$ & 4.18 & 243 & $31.0 \pm 0.1 \mathrm{c}$ & 1.03 & 4.08 & 1.16 \\
\hline $\mathrm{H}_{2}$ & 600 & $74.0 \pm 4.1$ & 34 & $75.9 \pm 2.7 \mathrm{c}$ & 4.33 & 219 & $30.4 \pm 0.1 \mathrm{~b}$ & 1.00 & 4.32 & 1.23 \\
\hline
\end{tabular}

Within the same column, there is a significant difference $(\mathrm{P}<0.05$, Tukey's test $)$ between values followed by a different letter. Mean \pm SE. F, fecundity (number of larvae emerged from the eggs laid during the egg laying period); DT, development time (in days); $\mathrm{B}_{\mathrm{P}}$, biotic potential; $\mathrm{DT}_{\mathrm{r}}$, ratio between the development time of the considered strain by the one of the susceptible strain; $\mathrm{C}_{\mathrm{BP}}$, ratio of the biotic potential between the considered strain and the susceptible strain.

Our results confirm those of Beeman and Nanis (1986) who showed a stability of $R^{\text {mal }}$ allele in Tribo-lium laboratory populations over six generations in the absence of malathion. However, these studies did not report estimates of fitness and selection coefficient values. Our fitness estimates show that the proportion of malathionspecific resistant individuals increases slightly in populations of T. castaneum during 10 generations in the absence of insecticide. The homozygous and heterozygous resistant strains of T. castaneum have a higher fitness than the homozygous susceptible strain. As reported for other species and other resistance mechanisms (Roush and McKenzie 1987, McKenzie 1996 and references therein), in T. castaneum, malathion-specific resistance is not associated with reduced fitness. In T. castaneum, our $\mathrm{B}_{\mathrm{P}}$ study shows that developmental stages of the susceptible homozygous strain develop at a slightly faster rate than those of the resistant heterozygous and homozygous strains. However, lower fecundity of susceptible insects is likely to lead to overall lower population growth rates than in the resistant strains. As suggested by Dyte (1990), malathion-specific resistance in T. castaneum does not involve any loss of fitness under laboratory or field conditions.

Dieldrin, diazinon, and malathion-resistant strains of the sheep blowfly Lucilia cuprina showed reduced fitness compared with susceptible strains when resistance has first evolved. However, due to the continued use of diazinon, besides the development of resistance, a fitness modifier was selected. Modified dia-zinon-resistant phenotypes show similar developmental stability and relative fitness compared with susceptible insects (McKenzie 1993). In T. castaneum, the malathion-specific resistance gene(s) has no pleiotropic fitness costs. Because fitness costs have never been noted in malathion-specific resistant T. castaneum populations, it seems that there has been no coadaptation with a fitness gene modifier or that the fitness modifier is closely linked to the malathion-specific resistance gene (s). In T. castaneum, resistance allele fitness studies have shown that the resistant allele had no major fitness costs (Beeman and Nanis 1986). Our results combined with those of Beeman and Nanis (1986) suggest that in T. castaneum the fitness of malathion-resistant insects is not dependent on their genetic background. A similar independence of fitness and genetic background also was observed in L. cuprina and dieldrin resistance (McKenzie 1996) and Musca domestica and diazinon resistance (Whitehead et al. 1985).

When a single gene is involved in insecticide resistance, the fitness of the heterozygous relative to the susceptible homozygous is critical because the resistance gene is carried primarily as the heterozygous when the gene is at low frequency during the early stages of resistance (Roush and Plapp 1982). We observed that the two heterozygous malathion-specific resistant strains had a heterosis advantage that confers on them the best fitness. Resistant heterozygous insects therefore have a double selective advantage. In addition to a better fitness than homozygous susceptible insects, they also can tolerate high malathion concentrations.

Because selection will not reduce the frequency of resistant genes by acting against resistant phenotypes when the corresponding insecticide is not being used, chemical control strategies based on the relative fitness disadvantage of insecticide-resistant insects will not succeed for malathion-specific resistance in T. castaneum. However, White and Bell (1988), regarding the vulnerability of the heterozygous first instars, postulated that resistance could be reversed by introducing susceptible insects within the population. Nevertheless, this method seems to be more effective with nonspecific malathion resistant strains (Wool and Manheim 1980) than with malathion-specific resistant T. castaneum strains (White and Bell 1988). 
Because world trade in cereals implies the circulation of freight from one storage place to another, it allows the dispersal of pests from different geographic areas and therefore of different genetic backgrounds and selective life histories. This will sometimes result in the introduction of resistant phenotypes in silos already contaminated with susceptible insects. Because genetic background does not influence fitness in the case of malathion-specific resistance and that no fitness disadvantage accompanies resistance genotypes in insects, the dispersal of resistant individuals will result in the development of malathion-specific resistance in the untreated populations. This may finally result in the failure of control of these populations with malathion.

\section{Acknowledgments}

We thank J. C. Gilson and F. Pierreux for technical assistance; P. Golob (Natural Resource Institute, Chatham, UK) and F. Fleurat-Lessard (Institut National de la Recherche Agronomique, Bordeaux, France) for providing the two T. castaneum strains; and G. Pierrard and J. A. McKenzie for their comments on the manuscript. This work was funded by the Fonds pour la Formation à la Recherche dans l'Industrie et dans l'Agriculturre to L. A. We also thank the two reviewers for their helpful comments.

\section{References}

ArgentineJ.A.,J.M.Clarck,and D.N.Ferro. 1989. Relative fitness of insecticide resistant Colorado potato beetle strains (Coleoptera: Chrysomelidae). Environ. Entomol. 18: 705-710.

Beeman, R. W. 1983. Inheritance and linkage of malathion resistance in the red flour beetle. J. Econ. Entomol. 76: 737-740.

Beeman, R. W., and S. M. Nanis. 1986. Malathion resistance alleles and their fitness in the red flour beetle (Co-leoptera: Tenebrionidae). J. Econ. Entomol. 79: 580-587.

Bourguet, D., T. Lenormand, T. Guillemaud, V. Marcel, D. Fournier, and R. Raymond. 1997. Variation of dominance of newly arisen adaptive genes. Genetics 147:1225-1234.

Champ, B. R. 1984. Pesticide resistance in stored product insects, pp. 681-690. In B. R. Champ [ed. ], Proceedings of the Australian Development Assisting Course on Preservation of Stored Cereals, Canberra, Australia, 1981.

Champ, B. R., and C. E. Dyte. 1976. Report of the FAO global survey of pesticides susceptibility of stored grain pests. Plant production and protection. Food and Agriculture Organization of the United Nations, series 5. FAO, Rome.

Clarke, B., and J. Murray. 1962. Changes in gene-frequency in Cepaea nemoralis (L.): the estimation of selective values. Heredity 17: 467476.

Cochran, D.G. 1993. Decline of pyrethroid resistance in the absence of selection pressure in a population of German cockroaches (Dictyoptera: Blattelidae). J. Econ. Entomol. 86: 1639-1644.

Dyte, C. E. 1990. Living with resistant strains of storage pests, pp. 947-959. In F. Fleurat-Lessard and P. Ducom [eds. ], Proceedings, 5th International Working Conference of Stored-Product Protection. Bordeaux, France.

Falconer, D. S. 1960. Introduction à la génétique quantitative. Masson et Cie, Paris, France.

Ferrari, J. A., and G. P. Georghiou. 1981. Effects on insec-tidal selection and treatment on reproductive potential of resistant, susceptible, and heterozygous strains of the southern house mosquito. J. Econ. Entomol. 74: 323-327.

Georghiou, G P. 1983. Management of resistance in arthropods. Plenum, New York.

Haubruge, E., L. Arnaud, and J. Mignon. 1997. The impact of sperm precedence in malathion resistance transmission in population of the red flour beetle Tribolium cas-taneum (Herbst) (Coleoptera, Tenebrionidae). J. Stored Prod. Res. 33: 143-146.

McKenzie, J. A. 1993. Measuring fitness and intergenic interactions: the evolution of resistance to diazinon in Lu-cilia cuprina. Genetica 90: 227-237.

McKenzie, J. A. 1994. Selection at the diazinon resistance locus in overwintering populations of Lucilia cuprina (the Australian sheep blowfly). Heredity 73: 57-64.

McKenzie, J. A. 1996. Ecological and evolutionary aspects of insecticide resistance. R.G Landes, Austin, TX.

McKenzie, J. A., and K O'Farrell. 1993. Modification of developmental instability and fitness:malathion-resistance in the Australian sheep blowfly, Lucilia cuprina. Genetica 89: 67-76.

McKenzie, J. A., and M. J. Whitten. 1982. Selection for insecticide resistance in the Australian sheep blow fly. Ex-perientia38: 84-85. 
Published in: Journal of economic entomology (2001), vol. 94, iss. 2, pp. 552-557

Status: Postprint (Author's version)

Minitab. 1998. User's manual, version 12.2 for Window. Minitab, Minitab, State College, PA.

Muggleton, J. 1983. Relative fitness of malathion-resistant phenotypes of Oryzaephilus surinamensis L. (Coleoptera: Silvanidae). J. Appl. Ecol. 20: 245-254.

Muggleton, J. 1986. Selection for malathion resistance in Oryzaephilus surinamensis (L.) (Coleoptera: Silvanidae): fitness values of resistant and susceptible phenotypes and their inclusion in a general model describing the spread of resistance. Bull. Entomol. Res. 76: 469-480.

Parello,M. P., and J. T. Trumble. 1989. Decline in resistance in Liriomyza trifolii (Diptera: Agromyzidae) in the absence of insecticide selection pressure. J. Econ. Entomol. 82: 365-368.

Parkin, E. A, E.I.C. Scott, and R. Forester. 1962. Increase resistance of stored-product insects to insecticides. The resistance of field strains of beetles, (c) Tribolium casta-neum. Pest Infest. Res. 1961: 34-35.

Raymond, M. 1993. PROBIT CNRS-UMII. Licence L93019. Avenix G. Prato and A. Ratsira, 34680 St. George d'Orques, France.

Roush, R. T., and M. A. Hoy. 1981. Laboratory, glasshouse, and field studies of artificially selected carbaryl resistance in Metaseiulus occidentalis. J. Econ. Entomol. 74:142-147.

Roush, R. T., and J. A. McKenzie. 1987. Ecological genetics of insecticide and acaricide resistance. Annu. Rev. Entomol. 32: 361-380.

Roush, R. T., and F. W. Plapp. 1982. Effects of insecticide resistance on biotic potential of the house fly (Diptera: Muscidae). J. Econ. Entomol. 75: 708-713.

Spollen, K M., M. W. Johnson, and B. E. Tabashnik. 1995. Stability of fenvalerate resistance in the leafminer para-sitoid Diglyphus begini (Hymenoptera, Eulophidae). J. Econ. Entomol. 88: 192-197.

White, N.D.G, and R. J. Bell. 1988. Inheritance of malathion resistance in a strain of Tribolium castaneum (Co-leoptera:Tenebrionidae) and effects of resistance genotypes on fecundity and larval survival in malathion-treated wheat. J. Econ. Entomol. 81: 381-386.

White, N.D.G, and R. J. Bell. 1990. Relative fitness of malathion-resistant strain of Cryptolestes ferrugineus (Co-leoptera:Cucujidae) when development and oviposition occur in malathion-treated and untreated wheat kernels. J. St. Prod. Res. 26: 23-37.

White, N.D.G, and R J. Bell. 1995. A malathion resistance gene associated with increased life span of the rusty grain beetle, Cryptolestes ferrugineus (Coleoptera:Cucujidae). J. Gerontol. 50: 9-13.

Whitehead, J. R, R. T. Roush, and B. R. Norment. 1985. Resistance stability and coadaptation in diazinon-resis-tant house flies (Diptera: Muscidae). J. Econ. Entomol. 78: 25-29.

Wool, D., S. Noiman, O. Manheim, and E. Cohen. 1982. Malathion resistance in Tribolium strains and their hybrids: inheritance patterns and possible enzymatic mechanisms (Coleoptera, Tenebrionidae). Biochem. Gene 20: 621-636.

Wool, D., and O. Manheim. 1980. Genetically-induced susceptibility to malathion in Tribolium castaneum despite selection for resistance. Entomol. Exp. Appl. 28: 183-190. 